\title{
Effect of Recycling on the Rheological, Mechanical and Optical Properties of Polycarbonate
}

\author{
Ferenc Ronkay \\ Department of Polymer Engineering \\ Budapest University of Technology and Economics \\ Müegyetem rkp. 3, H-1111 Budapest, Budapest, Hungary \\ e-mail: ronkay@pt.bme.hu
}

\begin{abstract}
The research was aimed at analyzing the polycarbonate scrap arising during production and its possible secondary utilization. The analysis of morphological, rheological and thermal data revealed significant differences between the original pellets and the reground material obtained from injection molded parts. Test specimens were injection molded from various mixtures of the virgin and the reground material, and their mechanical and physical properties were analyzed. Based on the results the reground material may be used in less than 20\% proportion, as the mechanical properties of the products do not deviate significantly from those of the products made from virgin polycarbonate.
\end{abstract}

Keywords: polycarbonates; recycling; mechanical properties; optical properties; morphology

\section{Introduction}

In recent years, reprocessing of polymers has been widely used in plastics converting industries [1-3]. It is connected to the increasing awareness of environmental issues, to the desire to save resources, and to the high levels of scrap material generated during plastics conversion. There is a high demand for the recycling of scraps considering the relatively high cost of polymer production. To solve this problem the recycling of scrap material, and mixing it with virgin material, is the most common solution [4-5].

Polycarbonate (PC) is one of the important engineering plastics with a wide variety of applications due to the excellent mechanical properties, high impact strength, heat resistance and high modulus of elasticity, as well as due to its excellent balance of toughness, clarity, high thermal resistance and transparency 
[6-7]. The recycling of this plastic material after the end of first life cycle has attracted attention recently [8-9].

Some physical and mechanical properties of PC can be severely reduced by recycling. Pérez at al. reported that after ten times of recycling the tensile strength reduced by $30 \%$ [10].

The rheological analysis of the dilute solution of macromolecular materials can give more information about the size and shape distribution of macromolecules. During the repetitive injection molding of polycarbonate, the molecular mass changes were studied by observing a rapid decrease in molecular weight, explained by two simultaneous degradation mechanisms [11].

In recent years the degradation of PC during accelerated aging tests has been studied by several researchers, among them the durability and a predictability of the properties to cover the whole lifecycle of the PC, as well as the degradation mechanism which occurs at the molecular level [12].

Long and Sokol studied the effect of moisture on the degradation of polycarbonate during injection molding [13]. It has been shown that even low moisture content during processing adversely affects the mechanical properties of the final product.

The effect of recycling on the properties of injection molded polycarbonate was studied by Shea and Nelson, who evaluated the extent of degradation by measuring melt flow rate, impact strength and molecular weight [14]. After ten times of recycling the value of melt flow rate increased five times.

Other works focused on the transparency of PC materials influenced by UV irradiation. The effect of UV irradiation was investigated on the structure and optical properties of polycarbonate material, and it was found that irradiation leads to a decrease of the optical energy gap of PC; they concluded that the decrease in optical energy gap could be due to the photo-degradation of $\mathrm{PC}$ and the formation of defects and clusters in the material [15].

The aim of the present study was to analyze the great amount of polycarbonate waste which arises during polymer processing and to find possibilities for secondary utilization. The aim was to determine the optimal rate of the recycled material as well, where the properties of the final product are still acceptable in respect of mechanical and optical properties, compared to the original polycarbonate product. 


\section{Experimental Work}

\subsection{Materials and Processing}

Makrolon 1804 (Bayer) polycarbonate was used as virgin material in our study, and the reground obtained from the scrap of injection molded parts made of the same grade was used as recyclate. The ratio of the reground material to the original PC was changed from 0 to $100 \%$.

Dumb-bell type test specimens were injection molded for the mechanical tests from the virgin material, from the reground recyclate and from various mixtures of the two. Injection molding was performed on an Arburg Allrounder 320C 600-250 injection molding machine. The zone temperatures were as follows from the feeding zone to the nozzle: $275 / 285 / 290 / 295 / 300^{\circ} \mathrm{C}$. The mold temperature was $80^{\circ} \mathrm{C}$, and the injection pressure was 1000 bar. In order to prevent hydrolytic degradation, the materials were dried before melting at $120^{\circ} \mathrm{C}$ for 4 hours.

\subsection{Characterization Methods}

Viscometric parameter determination was carried out at $25 \pm 0.1^{\circ} \mathrm{C}$, in chloroform solution, using an Ubbelohde $\mathrm{OB}$ viscosimeter. For viscosity-average molecular weight determination, constants $\mathrm{K}=0.012 \mathrm{~cm}^{3} / \mathrm{g}$ and $\mathrm{a}=0.82$ were employed [1617].

Melt flow rate was measured on CEAST Melt Flow Modular Line equipment at $270^{\circ} \mathrm{C}$ with $2.16 \mathrm{~kg}$ load. The materials were dried before measurements at $120^{\circ} \mathrm{C}$ for different periods between 0 and 220 minutes.

In order to determine the glass transition temperature, DMA tests were made using Perkin Elmer DMA 7 type equipment in displacement-controlled mode, with 10 $\mu \mathrm{m}$ amplitude, at $1 \mathrm{~Hz}$ frequency. Three point bending mode was used for excitation.

Tensile tests were performed according to the EN ISO 527 standard using a ZWICK Z020 type universal tensile tester at a deformation rate of $20 \mathrm{~mm} / \mathrm{min}$, at room temperature.

Charpy impact tests were performed using CEAST Resil Impactor Junior type equipment with $15 \mathrm{~J}$ impact energy, a $20^{\circ}$ starting angle and $0.589 \mathrm{~m} / \mathrm{s}$ impact velocity.

Transmission optical tests were made with a JASCO V-530 UV/VIS type spectrometer. Prior to these tests, the surface of the test specimens was polished to an average of $1 \mu \mathrm{m}$ roughness, using a Struers type polishing machine. 


\section{Results and Discussion}

\subsection{Changes of the Average Molecular Mass}

Changes of the viscosity average molecular mass at various levels of processing are shown in Fig. 1. It can be observed that the virgin pellet exhibits the highest average molecular mass $(18,000 \mathrm{~g} / \mathrm{mol})$; that of the reground is smaller $(16,500$ $\mathrm{g} / \mathrm{mol})$.

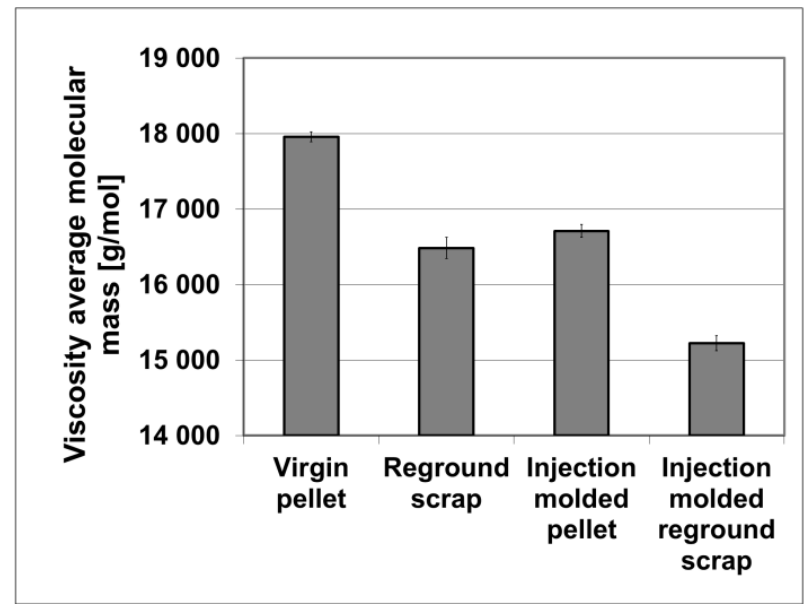

Figure 1

Viscosity average molecular mass of polycarbonates processed and reprocessed to various degrees

It is caused by the strong degrading effect of the shear forces and by the thermal impact encountered during injection molding. The average molecular mass of the test specimens injection molded from the virgin pellets was found to be similar $(16,700 \mathrm{~g} / \mathrm{mol})$, the slight difference might be due the minor differences between the injection molding parameters used for preparing the test specimens and the parts. The lowest value $(15,200 \mathrm{~g} / \mathrm{mol})$ was measured on test specimens injection molded from the regrind. By now the material has undergone two processing cycles, so the molecular chains are degraded to a higher degree. It can be established that the average molecular mass of the processed material decreases by about $8 \%$, and that of reprocessed material by about $15 \%$.

\subsection{Changes in Melt Viscosity}

The melt flow rates of the virgin pellet and of the recycled regrind were studied as a function of the drying time. The results are shown in Fig. 2. It can be observed that the flow rate of the recycled material without drying is higher (recyclate: $21.8 \mathrm{~cm}^{3} / 10 \mathrm{~min}$; virgin: $17.5 \mathrm{~cm}^{3} / 10 \mathrm{~min}$ ), and this difference persists after drying. 


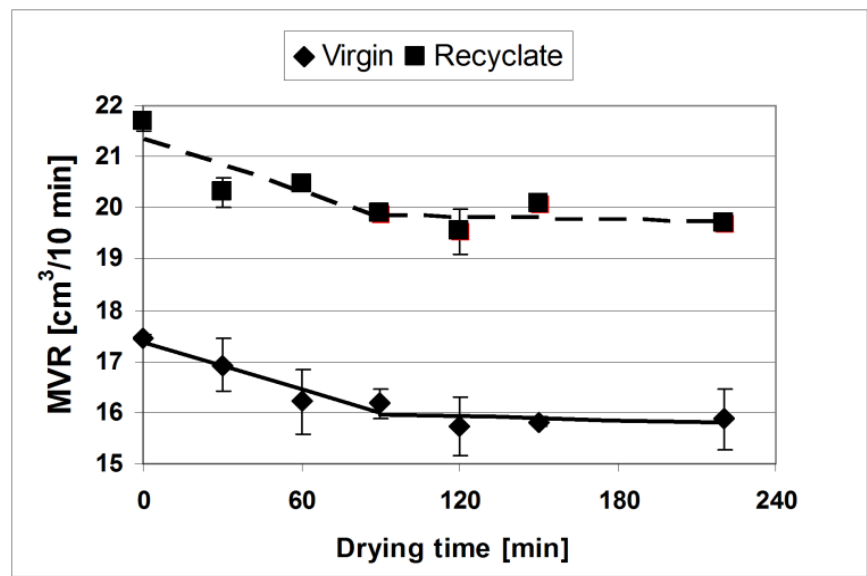

Figure 2

Volumetric melt flow rate of the virgin pellets and of the reground recyclate as a function of the drying time (drying temperature: $120^{\circ} \mathrm{C}$ )

The melt flow rate of both materials stabilized and became constant after about two hours of drying (recyclate: $19.8 \mathrm{~cm}^{3} / 10 \mathrm{~min}$; virgin: $15.8 \mathrm{~cm}^{3} / 10 \mathrm{~min}$ ), i.e. they exhibited similar behavior during drying. The difference can be explained by the degradation of the molecular chain: in the reground recyclate, shorter molecular chains can be found, which hider the melt flow to a lesser degree. The melt flow rate of the reground recyclate is about $25 \%$ higher than that of the virgin material.

\subsection{Changes in the Thermal Properties}

The glass transition determined by DMA is ascribed to the maximum temperature of the mechanical loss (see Fig. 3). It can be observed that the glass transition temperature of the virgin material $\left(135.8^{\circ} \mathrm{C}\right)$ is $1.1^{\circ} \mathrm{C}$ higher than that of the recycled material $\left(134.7^{\circ} \mathrm{C}\right)$.

This shift can be explained by the shortening of the molecular chains: the movement of shorter chains' segments starts at lower temperature. 


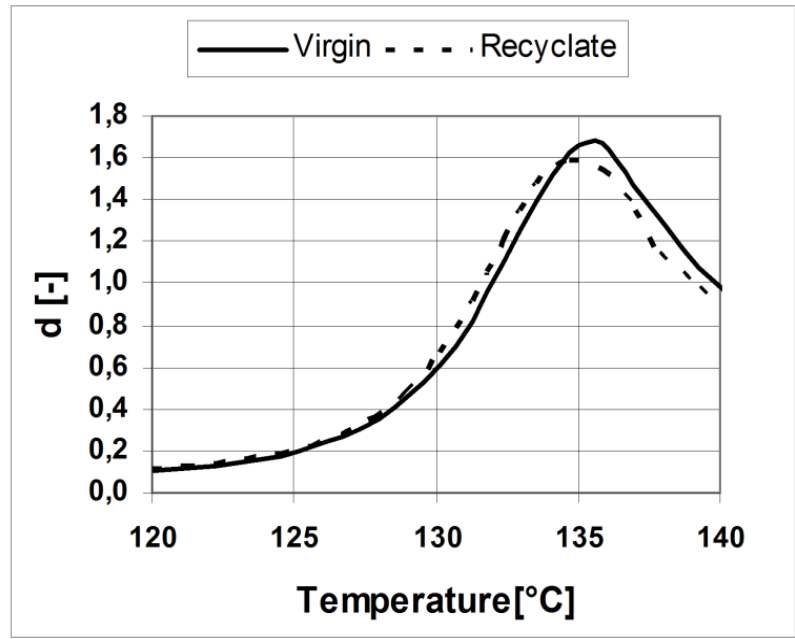

Figure 3

Change of the mechanical loss factor

\subsection{Changes in the Mechanical Properties}

\subsubsection{Changes in the Tensile Strength}

The dependence of the tensile strength on the amount of recycled material is shown in Fig. 4. Analyzing the plot it can be concluded that the value of the tensile strength increases slightly with the recycled material content. This increase is not significant: only $1-2 \%$ with respect to the virgin material.

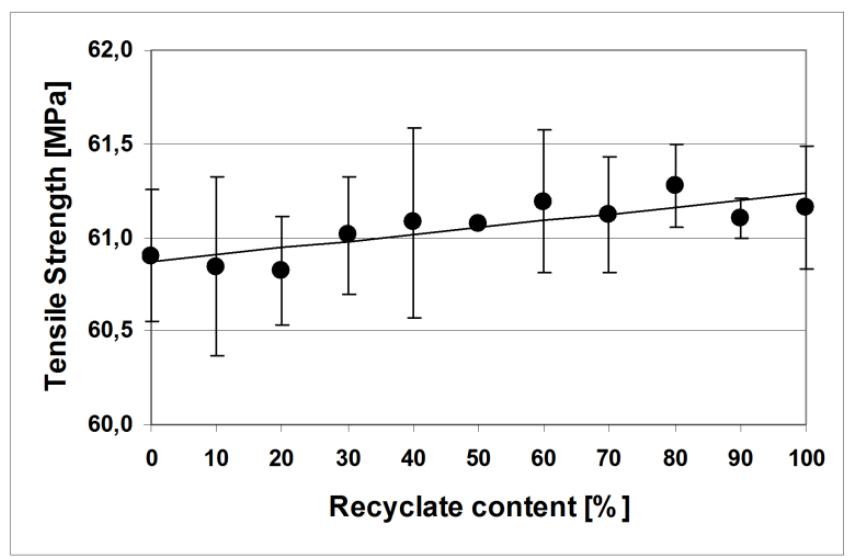

Figure 4

The tensile strength of the material as a function of the recyclate content 
This trend seems to contradict somewhat earlier literature findings [18], according to which a decreasing molecular mass results in decreasing strength (according to equation 1 ).

$\sigma_{\text {recycled }}=\sigma_{\text {virgin }}\left(1-\frac{M_{\text {difference }}}{M_{\text {virgin }}}\right)$

where $\sigma_{\text {recycled }}$ is the expected strength of the recycled material, $\sigma_{\text {virgin }}$ is the strength of the original (virgin) material, $\mathbf{M}_{\text {difference }}$ is the decrease of the average molecular mass, and $\mathbf{M}_{\text {virgin }}$ is the average molecular mass of the original (virgin) material.

Using equation (1) in our case, one would expect a $9 \%$ decrease between the strength of the recycled material and of the virgin material (equation 2).

$\sigma_{\text {recycled }}=60,9 M P a\left(1-\frac{16700-15200}{16700}\right)=60,9 M P a * 0,91=55,4 M P a$

The slight strength improvement observed in the tests may be due to the changes in the orientation of the amorphous macromolecular chains. Shorter chains may orient easier during injection molding along their long axis; thus they can bear more load during the tensile test. The prerequisite of this slight improvement is that the material be absolutely free of all contaminations, as even a small amount of contamination would serve as a defect site, which would decrease the strength.

\subsubsection{Changes in the Tensile Modulus}

The tendency of the change of the tensile modulus is similar that of the tensile strength (see Fig. 5).

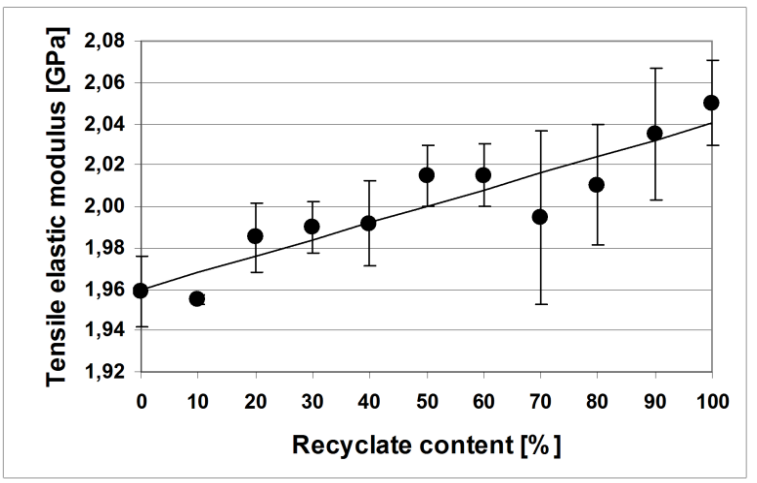

Figure 5

The tensile modulus of the material as a function of the recyclate content

If compared to the modulus of the virgin material $(1.96 \mathrm{GPa})$, the modulus of the recyclate increased slightly, by $4.5 \%$. It can be established that the rigidity of the material increases with the recyclate content, but the improvement is insignificant. 


\subsubsection{Changes in the Elongation at Break Values}

Elongation at break values measured during the tensile tests are shown in Fig. 6 .

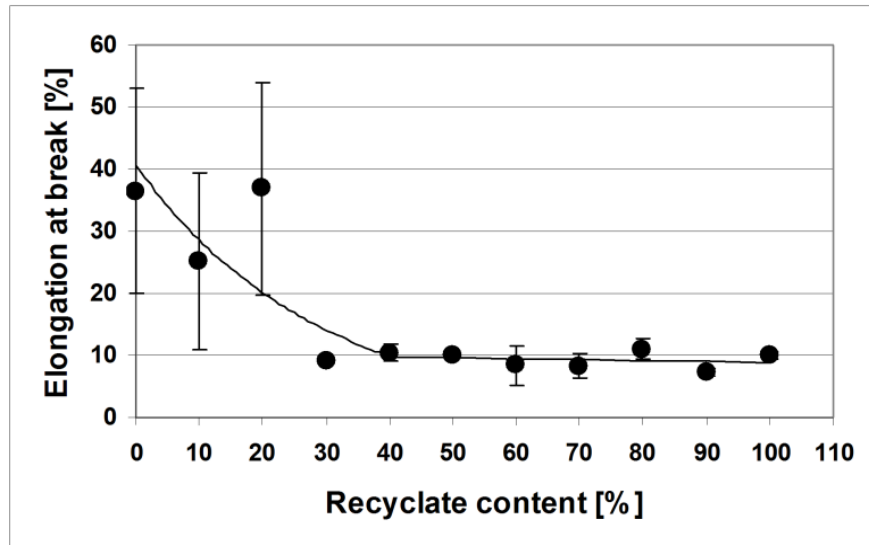

Figure 6

Change of the elongation at break as a function of the recyclate content

The elongation at break decreased significantly with up to $30 \%$ recyclate content. The decrease was about $75 \%$ with respect to the virgin material. The elongation at break is very sensitive to the change of the average molecular mass. The elongation at break of the virgin material and of the samples containing $10 \%$ or $20 \%$ recyclate is $25-40 \%$ of the original length, although the results exhibit fairly large scatter. In the range of $30-100 \%$ recyclate, content the elongation at break is only $10 \%$ of the original length. In this range there is no significant change and the scatter around the average is also smaller.

The large drop in the elongation at break in the samples containing 30-100\% recyclate affects the quality and usefulness of the produced parts (e.g. snap-fit closures), and therefore it is not recommended to use more than $20 \%$ reground recyclate.

\subsubsection{Changes in the Impact Strength}

Values of the impact strength calculated from the flexural impact test are shown in Fig. 7.

Decreasing impact strength values can be observed with increasing recyclate content. The impact strength of the test specimens injection molded from pure recyclate is $12 \%$ lower than that of the test specimen produced from the virgin material. Test results agree with the elastic modulus measured in the tensile test: with increasing recyclate content the material becomes stiffer, and its ductility decreases. 


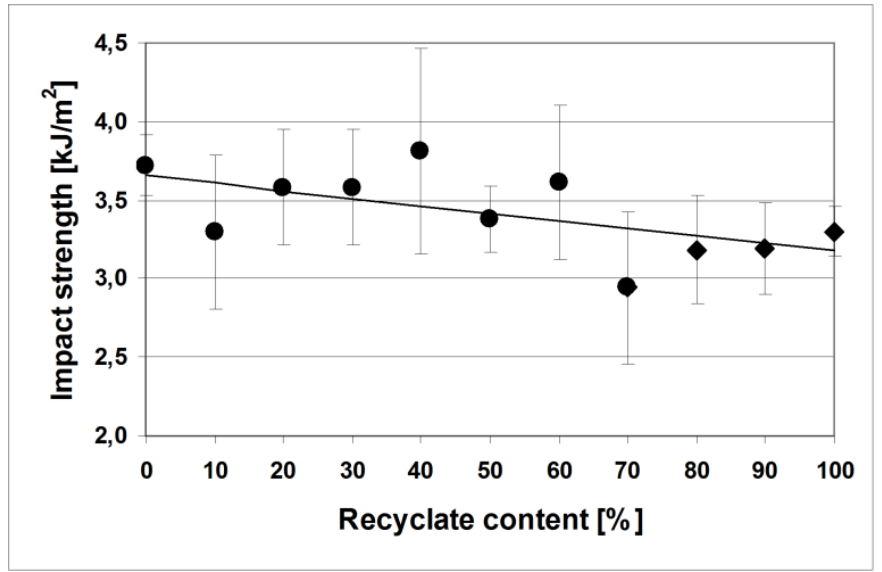

Figure 7

Impact strength as a function of the recyclate content

\subsection{Changes in the Optical Properties}

Light transmittance of the virgin and recycled material is shown in Fig. 8. The transmittance curves exhibit a similar character in both cases: in the 380-408 nm range (belonging to the violet color) the materials transmit less light, but in the $408-760 \mathrm{~nm}$ range they transmit $80-90 \%$ of the light, so they can be regarded as transparent. It can be observed that in the 380-408 $\mathrm{nm}$ range the transmittance of the virgin and of the recycled material differs significantly: the original transmits better short wavelength (violet) rays.

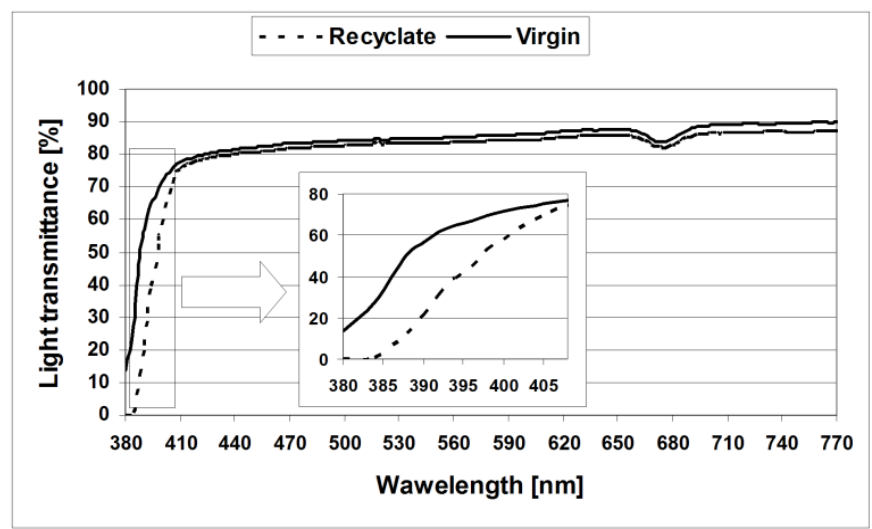

Figure 8

Light transmittance of the virgin and of the recycled test specimens as a function of the wavelength 
If the full visible light spectrum (white light) is transmitted through a transparent material, the complementary color of the absorbed color will be amplified. The complementary color of violet is yellow; i.e., if the material absorbs more violet, it will appear more yellow to the human eye. The irradiation absorbance of polymers changes with the molecular weight reduction, not only in the UV range but also in the visible light range. In the case of polycarbonate, decreasing molecular mass causes higher UV absorption, which is presumably related to the increased number of end-groups. This phenomenon is similar to the way in which the photodegradation of PC occurs, whereby the absorption varies in the same way in the range of wavelength between 250-400 $\mathrm{nm}$ [15]. Based on our measurements, 10$20 \%$ recyclate content does not deteriorate too strongly the UV transmission; above this concentration, however, the changing transmittance may cause distortion if the material is colored (the ratio of transmitted light is $57 \%$ at $290 \mathrm{~nm}$ in the case of original PET; $22 \%$ in the case of recycled PET; and $46 \%$ in the case of original PET with $20 \%$ recyclate content).

\section{Conclusions}

The degradation of polycarbonate during processing and its effects on the mechanical and optical properties of the material have been studied. It has been shown that the average molecular mass of polycarbonate decreases by about $8 \%$ during the first injection moulding and the subsequent grinding. Based on our test results, this $8 \%$ decrease in the average molecular mass causes about a $25 \%$ increase in the melt flow rate.

Changes in the mechanical properties were monitored by measuring the tensile and flexural impact properties. Test specimens were injection molded from various mixtures of the virgin pellets and reground material, using $10 \%$ steps. It has been established that the tensile strength and the tensile elastic modulus does not change too greatly, but the elongation at break and the impact strength values decrease significantly. Based on these findings, one can say that the use of more than $20 \%$ reground recyclate results in significant deterioration of the mechanical properties (especially of the impact strength) of the material.

In the optical studies, the transmittance of mixtures of various composition were studied in the full visible frequency range. Significant differences were found only in the first half of the violet range, where the absorbance of the recycled material is higher than that of the virgin material. The absorption of the violet light from the whole visible spectrum renders the material yellow for the human eye. Based on the study, a recyclate content above $10 \%$ causes a detectable difference in the violet absorption, although the color difference could not be detected by the naked eye between specimens made of various mixtures of the virgin and of the recycled material.

Based on these results, it can be concluded that the admixture of more than $20 \%$ reground recyclate may deteriorate the mechanical and optical properties of the product significantly. 


\section{Acknowledgement}

This work is connected to the scientific program of the "Development of qualityoriented and harmonized $\mathrm{R}+\mathrm{D}+\mathrm{I}$ strategy and functional model at BME" project. This project is supported by the New Széchenyi Plan (Project ID:TÁMOP4.2.1/B-09/1/KMR-2010-0002).

\section{References}

[1] Al-Salem S. M., Lettieri P., Baeyens J. Recycling and Recovery Routes of Plastic Solid Waste (PSW): A Review. Waste Manage 2009; 29:2625-2643

[2] Pegoretti A., Kolarik J., Slouf M. Phase Structure and Tensile Creep of Recycled Poly(Ethylene Terephthalate)/Short Glass Fibers/Impact Modifier Ternary Composites. Express Polym Lett 2009; 3:235-244

[3] Huiting S., Pugh R. J., Forssberg E. A Review of Plastics Waste Recycling and the Flotation of Plastics. Conserv Recy 1999; 25:85-109

[4] Saraiva Sanchez E. M. Ageing of PC/PBT Blend: Mechanical Properties and Recycling Possibility. Polym Test 2007; 26:378-387

[5] Eguiazabal J. I., Nazabal J. Effect of Reprocessing on the Properties of Bisphenol-A Polycarbonate. Eur Polym J 1989; 25:891-893

[6] Krawczak P. Plastics' Key Role in Energy-Efficient Building. Express Polym Lett 2009; 3:752

[7] Cao K., Ma X., Zhang B., Wang Y., Wang Y. Tensile Behavior of Polycarbonate over a Wide Range of Strain Rates. Mat Sci Eng A-Struc. 2010; 527:4056-4060

[8] Kahlen S., Wallner G. M., Lang R. W. Aging Behavior and Lifetime Modeling for Polycarbonate. Sol. Energy 2010; 84:755-759

[9] Krivtsov V., Wagner P. A., Dacombe P., Gilgen P. W., Haven S., Hilty L. M. Analysis of Energy Footprints Associated with Recycling of Glass and Plastic - Case Studies for Industrial Ecology. Ecol. Model. 2004; 174:175189

[10] Pérez J. M., Vilas J. L., Lazaa J. M., Arnáizb S., Mijangosa F., Bilbaoc E., Rodrígueza M., León L. M. Effect of Reprocessing and Accelerated Ageing on Thermal and Mechanical Polycarbonate Properties. J. Mater. Process. Tech. 2010; 210:727-733

[11] Glockner G. Polycarbonate Degradation under Processing Conditions. Plaste und Kautschuk, 1968; 15:632-635

[12] Weibin G., Shimin H., Minjiao Y., Long J., Dan Y. The Effects of Hydrothermal Aging on Properties and Structure of Bisphenol A Polycarbonate. Polym. Degrad. Stabil. 2009; 94:13-17 
[13] Long T. S., Sokol R. J. Molding Polycarbonate: Moisture Degradation Effect on Physical and Chemical Properties. Polym. Eng. Sci. 1974; $14: 817-822$

[14] Shea J. W., Nelson E. D., Cammons R. R. Effect of Recycling on the Properties of Injection Molded Polycarbonate. Techn. Pap.-Soc. Plast. Eng. $1975 ; 21: 614-617$

[15] Migahed M. D., Zidan H. M. Influence of UV-Irradiation on the Structure and Optical Properties of Polycarbonate Films. Curr. Appl. Phys. 2006; 6:91-96

[16] de Melo N. S., Weber R. P., Miguez Suarez J. S. Toughness Behavior of Gamma-irradiated Polycarbonate. Polym. Test. 2007; 26:315-322

[17] Lia C., Zhang Y., Zhanga Y., Zhangb C. Blends of Polycarbonate and Ethylene-1-Octylene Copolymer. Eur. Polym. J. 2003; 39:305-311

[18] Lawrence E. N., Robert F. L. "Mechanical Properties of Polymers and Composites" Marcel Dekker, New York 1994 